\title{
Parametric study of a microwave sensor dedicated to the dielectric spectroscopy of single particles and biological cells
}

\author{
W. Chen, D. Dubuc, K. Grenier \\ CNRS, LAAS, Univ. de Toulouse, 7 avenue du colonel Roche, F-31400 Toulouse, France
}

\begin{abstract}
This paper deals with a parametric study of a sensor dedicated to the microwave dielectric spectroscopy of individual particles or biological cells. The sensor is based on a coplanar topology with a capacitive gap and an embedded trap placed on top to precisely localize the particles or cells of interest. Various parameters such as the width of the capacitive gap, particle size and relative permittivity have been evaluated through simulations and validated with measurements. This study enables the optimization of the sensor in term of sensitivity.
\end{abstract}

Keywords- Sensor, microwave, dielectric spectroscopy, particles, biological cells, coplanar waveguide

\section{INTRODUCTION}

Applicative domains such as chemistry or biology require the routine use of characterization instruments able to analyze single particles or biological cells. Therefore, very efficient tools such as flow cytometers for instance have been developed and are mainly based on optical techniques. Electrical detection techniques may also be employed and have strongly been exploited in the past with the famous Coulter counter notably and its derivatives [1]-[2].

There is now an attractive interest to evaluate microwave dielectric spectroscopy as a new solution for particle and cellular analysis as it intrinsically presents numerous assets. Due to the frequency range, the cells may be measured directly in their culture medium, with miniature sensors compatible with lab-on-a-chip applications. Electromagnetic waves are also non invasive for the cells, without any requirement of preliminary cells preparation. The technique is also nondestructive, contact free and label free [3]. The counterpart of the technique is however its novelty. And one of its key issues is now to demonstrate that it is able to reliably detect single particles or biological cells. Wang et al. demonstrated in 2009 an interferometer centered at $5 \mathrm{GHz}$ able to discriminate the viability of yeast in water [4]. Ferrier et al. performed microwave measurements of single bead and cell in flow for cytometry purpose in 2010 [5]. In the team, has therefore been developed a microwave sensor able to detect one single bead or one single biological cell [6].

In order to optimize such a microwave sensor, we have performed a parametric study of the most important dimensions of the sensor or particle and dielectric properties. Results of such an investigation are indicated in this publication.

\section{DESCRIPTION OF THE MICROWAVE SENSOR DEDICATED TO THE MEASUREMENTS OF SINGLE PARTICLE OR CELL}

Figure 1 presents a three-dimensional view of the microwave sensor, obtained with the electromagnetic simulation software HFSS.

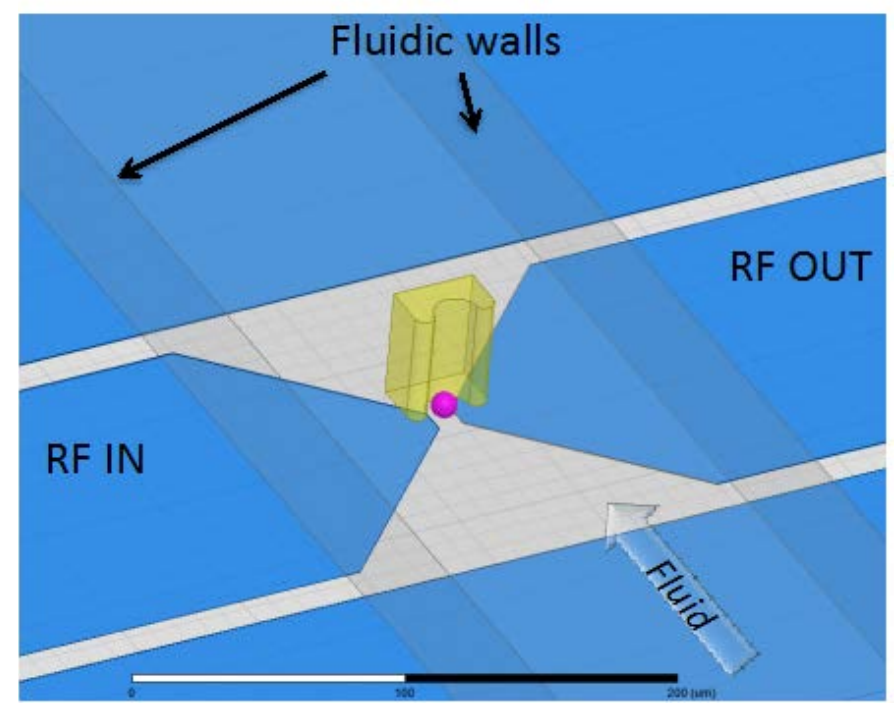

Fig. 1. 3D view of the sensor. The bead is indicated in pink at the bottom of a mechanical trap, presented here in yellow.

The sensor includes a coplanar waveguide on top of a quartz wafer. In the center of the structure is placed a capacitive gap, which exhibits a width of $10 \mu \mathrm{m}$. The central conductors placed apart have been narrowed in order to focalize the electromagnetic field in the capacitive gap [6].

On top of the coplanar waveguide is perpendicularly located a microfluidic channel, which conveys particles or biological cells in their liquid host medium. The solution used to stop and precisely localize the particle or cell on the capacitive gap corresponds to a trap, which is placed just above the gap, slightly shifted in order to have the particle centered on the capacitive gap. This mechanical trap exploits fluidic streamlines to first block a particle on the trajectory of the trap and then to divert any other particle [7]. The trap is part of the microfluidic channel and realized with a traditional material in microfluidics, the PDMS elastomer [8]. Figure 2 presents a photography of the center part of the microwave sensor. 


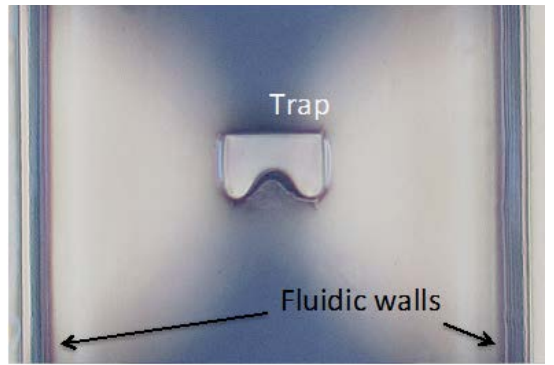

Fig. 2. Photography of the unloaded microwave sensor dedicated to single particle measurement.

Due to the height difference between the trap's top and the RF metallization, a clear view of both levels is not possible. The focus of the picture has been done on the blocker.

Both simulations and measurements of single particles have been performed with such a structure and are presented hereafter.

\section{VALIDATION OF THE ELECTROMAGNETIC SIMULATIONS OF SINGLE PARTICLES THROUGH MEASUREMENTS}

Before any parametric study, we looked at verifying and validating our finite elements simulations performed with HFSS and our extraction method of the dielectric contrasts. For this purpose, we have simulated a polystyrene bead with a diameter of $10 \mu \mathrm{m}$ over a $10 \mu \mathrm{m}$ wide capacitive gap. The bead was considered in De-Ionized (DI) water. In parallel, measurements of such a bead in a fabricated sensor have been performed. For the measurements, the sensor is connected through coplanar probes to a Vector Network Analyzer. The frequency range is ranging from $40 \mathrm{MHz}$ to $40 \mathrm{GHz}$. Dielectric contrasts have then been extracted with respect to the sensor loaded with only the host liquid medium, i.e. DI water. Further details on the extraction method may be found in [6].

Figure 3 and 4 present the corresponding results for both simulations and measurements.

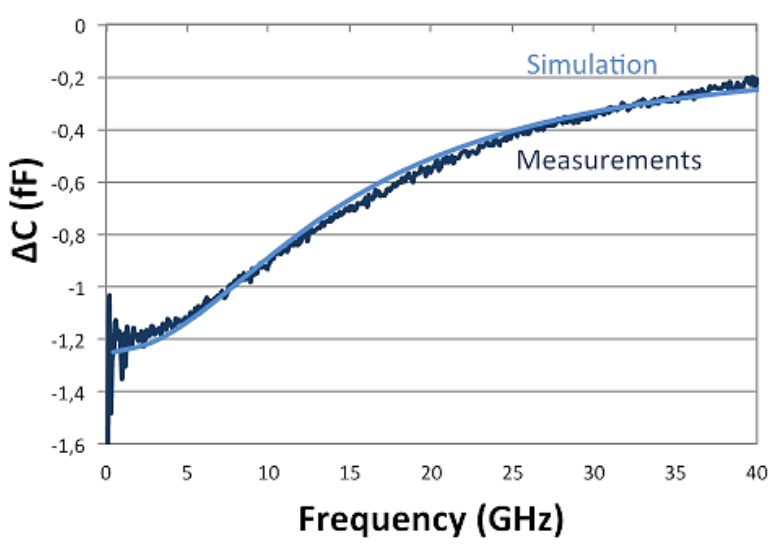

Fig. 3. Simulated and measured capacitive contrasts of a polystyrene bead with a diameter of $10 \mu \mathrm{m}$ on a capacitive gap of $10 \mu \mathrm{m}$, in DI water.

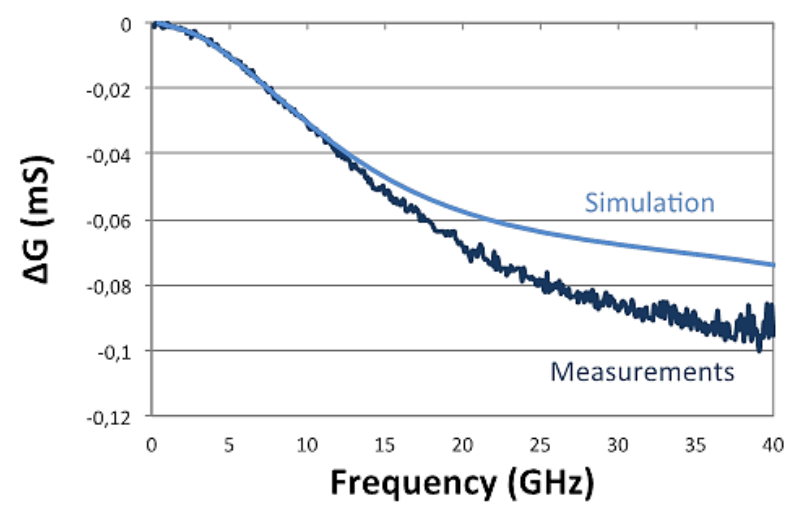

Fig. 4. Simulated and measured conductive contrasts of a polystyrene bead with a diameter of $10 \mu \mathrm{m}$ on a capacitive gap of $10 \mu \mathrm{m}$, in DI water.

A good fit between simulation and measurements is obtained for both capacitive and conductive contrasts. A difference between measurement and simulation appears on the conductive contrast above $15 \mathrm{GHz}$. It may be attributed to further losses induced by the host liquid medium, DI water, which is not pure as willing. Results are however quite similar and permit to validate our method of simulation.

\section{PARAMETRIC STUDY}

Based on the validation of our electromagnetic simulations, we have been able to conduct a parametric study on the single particle microwave sensor. Different parameters have been evaluated through simulations: the impact on the dielectric contrasts of the capacitive gap, the diameter of particles and their permittivity.

\section{A. Impact on the dielectric constrasts of the sensor capacitive} gap for a polystyrene bead of $10 \mu \mathrm{m}$ of diameter

First, variations on the capacitive gap size have been evaluated. The gap has been simulated with values ranging from 2 to $10 \mu \mathrm{m}$, while the diameter of the polystyrene bead is kept at $10 \mu \mathrm{m}$ in DI water. Fig. 5 presents the corresponding dielectric contrasts up to $40 \mathrm{GHz}$. For both contrasts (capacitive and conductive ones), the smaller gap leads to the larger dielectric contrasts. 

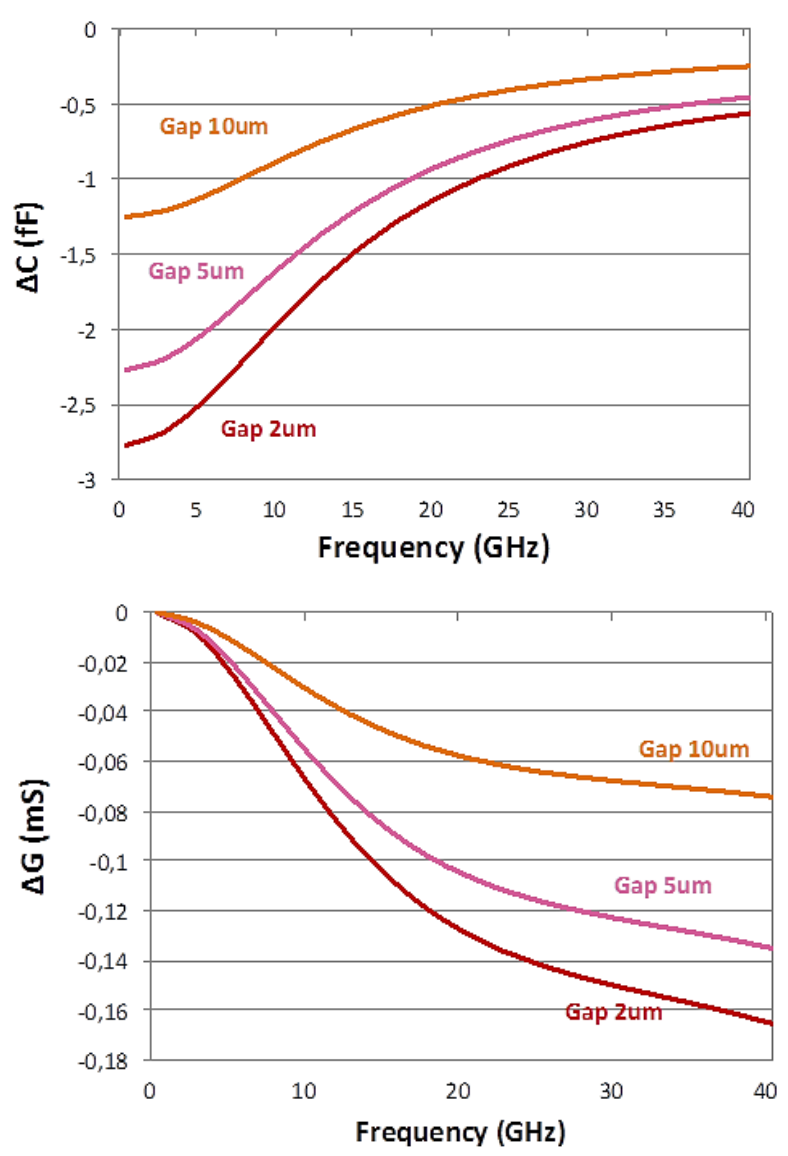

Fig. 5. Simulated capacitive and conductive contrasts of a polystyrene bead with a diameter of $10 \mu \mathrm{m}$ on top of a capacitive gap of 2,5 and $10 \mu \mathrm{m}$ wide, in DI water.

This result may be explained by the stronger focalization of the electromagnetic field inside the bead, when the gap width is minimized. Fig. 6 indicates the capacitive and conductive contrasts at 5 and $40 \mathrm{GHz}$ respectively versus the gap size. One may notice that the obtained variation may be considered as linear for both contrasts. A $2 \mu \mathrm{m}$ gap would be preferable to maximize the value of dielectric contrasts by a factor 2 . However, due to photolithographic and cost constraints during fabrication, a $5 \mu \mathrm{m}$ wide gap should be favored.

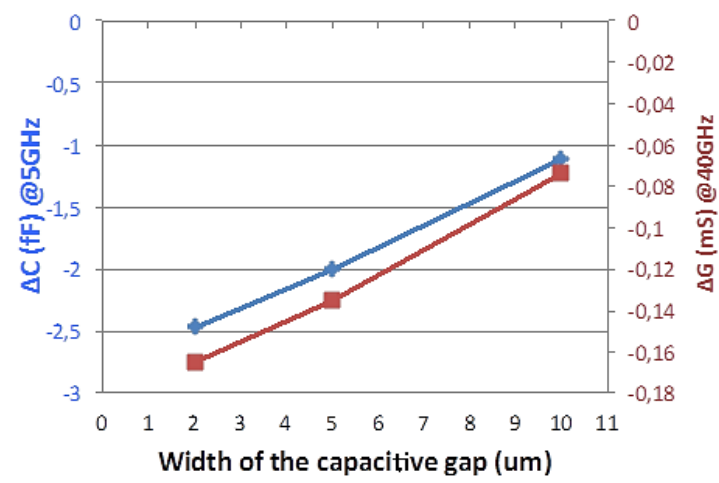

Fig. 6. Simulated capacitive and conductive contrasts of a $10 \mu \mathrm{m}$ diameter polystyrene bead versus the capacitive gap, in DI water.

\section{B. Impact of the diameter of polystyrene beads on the dielectric constrasts}

A similar study has also been performed depending on the diameter of beads. This time, the capacitive gap is maintained to $10 \mu \mathrm{m}$ large, whereas the diameter of bead is ranging from 10 to $20 \mu \mathrm{m}$. Simulated results are given in Fig. 7 and 8 .
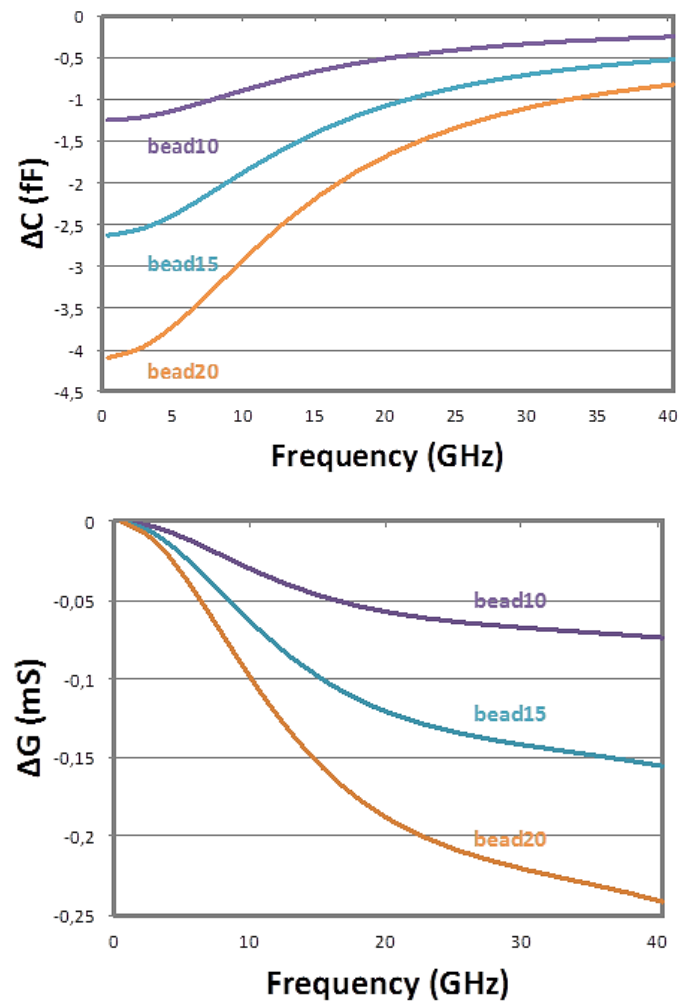

Fig. 7. Simulated capacitive and conductive contrasts of a polystyrene bead with a diameter of 10,15 and $20 \mu \mathrm{m}$ on top of a capacitive gap $10 \mu \mathrm{m}$ wide, in DI water.

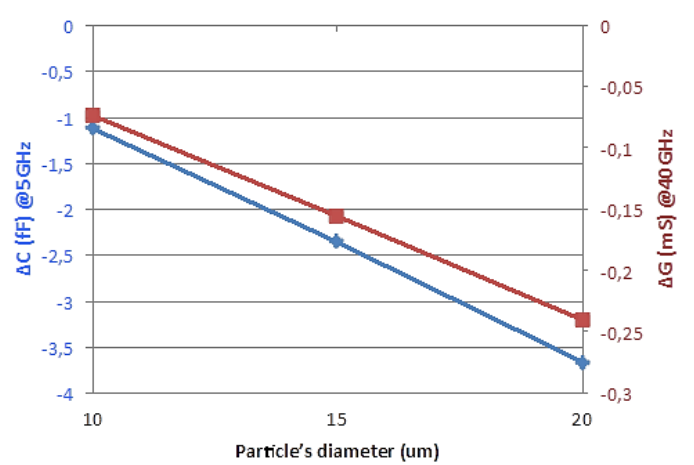

Fig. 8. Simulated capacitive and conductive contrasts versus the beads' diameter, in DI water.

As one may expect, the dielectric contrasts increases, as the size of the bead is larger. The capacitive contrast is enlarged by a factor higher than 3, for a diameter bead multiplied by 2 . More dielectric material is in interaction with 
the electromagnetic field, which leads to further detection of the bead under test. The contrasts variation versus particle diameter is also linear, as indicated in Fig. 8.

\section{Impact of the relative permittivity of beads on the dielectric constrasts}

Finally, the relative permittivity of a bead of $10 \mu \mathrm{m}$ of diameter has been modified during simulations, ranging from the fixed value of polystyrene, 2.5 , to 20 , which is close to water permittivity at $40 \mathrm{GHz}$. Simulated contrasts are given in Fig. 9.
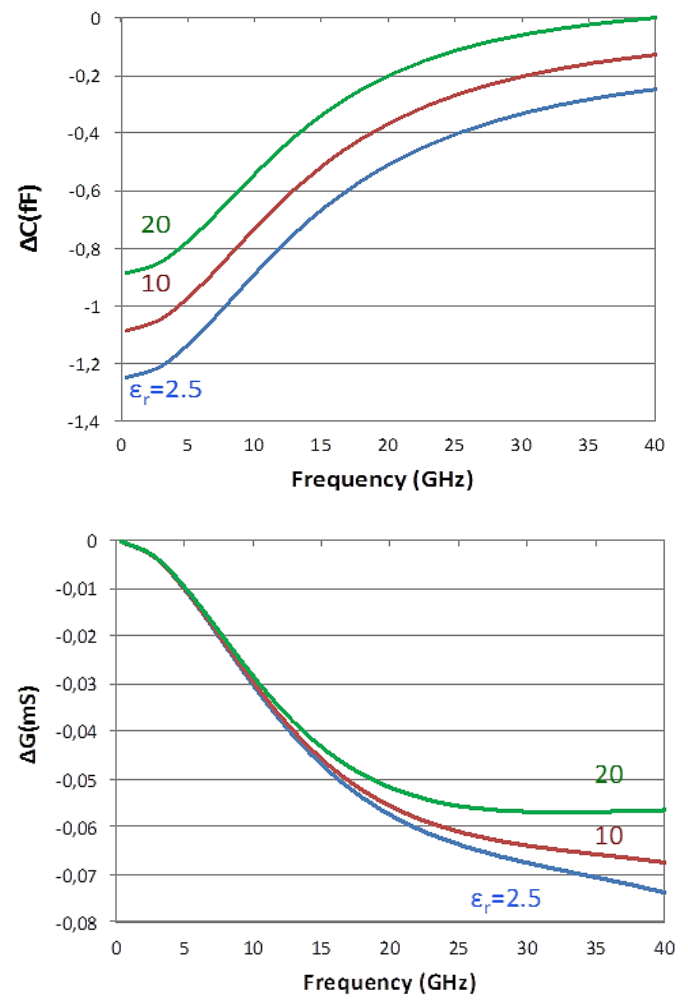

Fig. 9. Simulated capacitive and conductive contrasts for different relative permittivity of bead (from 2.5 to 20 ).

When the permittivity of the bead increases, the dielectric contrasts decreases. This is due to the fact that the host medium corresponds to water, which exhibits large values of permittivity versus frequency compare to polystyrene. As soon as the bead present a permittivity value closer to the host medium, the contrast are therefore minimized.

Relative permittivity variations impact mainly the capacitive contrast. Moreover, if the modification of permittivity is considered very important in term of value (attributed values from 2.5 to 20 constitutes very different dielectric materials), the related effect on the capacitive contrast remains small compare to the influence of the capacitive gap or the size of bead (see sections A and B).

\section{CONCLUSIONS}

A parametric study has been performed on a microwave sensor dedicated to the dielectric spectroscopy of single particle or biological cell. After the validation of the threedimensional simulations and dielectric contrasts extraction procedure through measurements, different dimensions have been evaluated. First, the width of the capacitive gap at the center of the sensor should therefore be lowered in order to enhance the sensitivity. Due to cost and easiness of fabrication considerations, a gap value of $5 \mu \mathrm{m}$ should be preferred. Secondly, any variation of the bead's diameter leads to a large modification of the two dielectric contrasts (capacitive and conductive ones). If the diameter is doubled, the capacitive contrast is almost multiplied by a factor 4. Finally, even particularly large modification of relative permittivity of the bead leads to weak dielectric contrasts variations (one third for a variation of 17.5).

\section{ACKNOWLEDGMENT}

The authors want to acknowledge the RENATCH network for supporting the microfabrication of the device in the LAASCNRS clean room.

\section{REFERENCES}

[1] T. Sun, H. Morgan, "Single-cell microfluidic impedance cytometry: a review," Microfluid. Nanofluid., vol. 8, pp. 423-443, 2010.

[2] D. Holmes, H. Morgan, "Single cell impedance cytometry for identification and counting of CD4 T-cells in human blood using impedance labels," Anal. Chem., vol. 82, pp. 1455-1461, 2010.

[3] K. Grenier, D. Dubuc, T. Chen, F. Artis, T. Chrétiennot, M. Poupot, J-J. Fournié, 'Recent Advances in Microwave-based Dielectric Spectroscopy at the Cellular Level for Cancer Investigations', invited paper in the Biomedical Applications of RF/Micro. Tech. Special Issue of IEEE TMTT, Vol. 61, Issue 5, Part 2, 2013, pp. 2023-2030

[4] Y. Yang, P. Wang et al., "Distinguishing the viability of a single yeast cell with an ultra-sensitive radio frequency sensor, Lab on a Chip, 10, pp. 553-555, 2010.

[5] G.A. Ferrier, S.F. Romanuik, D.J. Thomson, G.E. Bridges, M.R. Freeman, "A microwave interferometric system for simultaneous actuation and detection of single biological cells," Lab on a Chip, vol. 9, pp. 3406-3412, 2009.

[6] T. Chen et al, Microwave biosensor dedicated to the dielectric spectroscopy of a single alive biological cell in its culture medium, IEEE IMS (2013) 978-1-4673-2141.

[7] D. Di Carlo, N. Aghdam, L.P. Lee, "Single-cell enzyme concentrations, kinetics, and inhibition analysis using high-density hydrodynamic cell isolation arrays," Anal. Chem., vol. 78, pp. 4925-4930, 2006.

[8] G.M. Whitesides, "The origins and the future of microfluidics," Nature, vol. 442 , pp. $368-373,2006$ 